numerary bronchus supplying a segment of pulmonary tissue known as the tracheal lobe. ${ }^{4}$ In these cases bronchoscopy will show the normal position and trifurcation of the right upper lobe bronchus, allowing differentiation from the ectopic origin of a normal bronchus. For an accessory bronchus to be definitely designated as supernumerary it must be shown to supply lung parenchyma in excess of that normally encountered in the right upper lobe ${ }^{1}$ which has previously required bronchography. ${ }^{34}$ Tracheal lobes may lie in an extralobar or intralobar position and usually have a vascular supply from the pulmonary artery. ${ }^{4}$ It is uncertain whether lung parenchyma supplied by an abnormal bronchus is intrinsically more susceptible to disease, ${ }^{1}$ however, most patients with tracheal bronchi are asymptomatic and need no specific treatment. ${ }^{15}$ In some patients disease occurs in the lung supplied by a displaced or supernumerary bronchus, usually in the form of bronchiectasis. ${ }^{1}$ Surgical excision of the involved segment has been necessary in such patients. ${ }^{46}$

Our patient showed a true supernumerary tracheal bronchus that was distinguished from an ectopic bronchus by the normal anatomy of the right upper lobe bronchus. This case shows the ability of axial imaging to fully demonstrate a rare congenital abnormality of the bronchial tree. Computed tomographic scanning demonstrated the caudal course of the supernumerary bronchus and suggested the presence of the tracheal lobe, but was unable to define clearly the associated segment of lung. Magnetic resonance imaging showed the size and position of the tracheal lobe which, in this case, obviated the need for bronchography.

1 Ritsema GH. Ectopic right bronchus: indication for bronchography. $A \mathscr{F} R$ 1983;140:671-4.

2 McLaughlin FJ, Strieder DJ, Harris GBC, Vawter GP, Eraklis AJ. Tracheal bronchus: association with respiratory morbidity in childhood. $f$ Pediatr 1985;106:751-5.

3 Reed R, Cyr J, Marek J, Whitman G, Hoperman A. Bronchial anomaly of the right upper lobe. Ann Thorac Surg 1990; 50:980-1.

4 Rodgers BM, Harman PK, Johnson AM. Bronchopulmonary foregut malformations. The spectrum of anomalies. Ann Surg 1986;203:517-24.

5 Jackson GD, Littleton JT. Simultaneous occurrence of anomalous cardiac and tracheal bronchi: a case study. $₹$ Thorac Imag 1988;3:59-60.

6 Marks C. The ectopic tracheal bronchus: management of a child by excision and segmental pulmonary resection. $D$ is Chest 1966;50:652-4.

\section{Replacement of one lung by a large bulla in active tuberculosis}

\author{
Sulaiman A Al-Majed
}

A 70-year-old diabetic man with pulmonary tuberculosis developed a progressively enlarging bulla which occupied the whole left hemithorax and caused some shift of the mediastinum.

(Thorax 1995;50:427-428)

Keywords: tuberculosis, bulla, lung.

\section{Department of}

Medicine,

College of Medicine,

King Saud University,

PO Box 2925,

Riyadh 11461, Saudi

Arabia

S A Al-Majed

Reprint requests to:

Dr S A Al-Majed.

Received

22 December 1993

Returned to authors

21 February 1994

Revised version received

7 April 1994

Accepted for publication

9 June 1994
Large bullae are a known but rare complication of active tuberculosis. ${ }^{12}$ This is the first report of total replacement of one lung by a bulla which progressed in spite of successful chemotherapy.

\section{Case report}

A 70-year-old Saudi man was admitted to King coughing, yellow sputum, fever, and sweating for one month. Diabetes had been diagnosed four months previously and he was treated with Khalid University Hospital, Riyadh, with diet and an oral hypoglycaemic agent. He had never smoked and had no relevant occupational exposure. His chest symptoms persisted in spite of two courses of antibiotics. Chest radiography showed an infiltrate in the left mid zone without cavitation or elevation of the diaphragm. Bronchoscopic examination showed an inflamed, patent left main bronchus with multiple areas of ulceration. Abundant acid fast bacilli were seen in the bronchoalveolar lavage fluid. He was started on $300 \mathrm{mg}$ isoniazid, $600 \mathrm{mg}$ rifampicin, and $1.5 \mathrm{~g}$ pyrazinamide daily. Culture of lav-

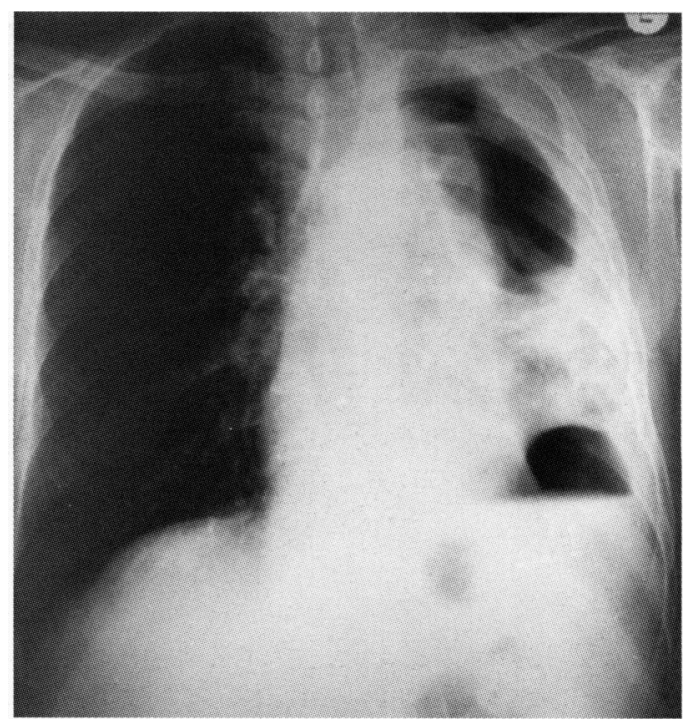

Figure 1 Chest radiograph showing two bullae in the left hemithorax. 


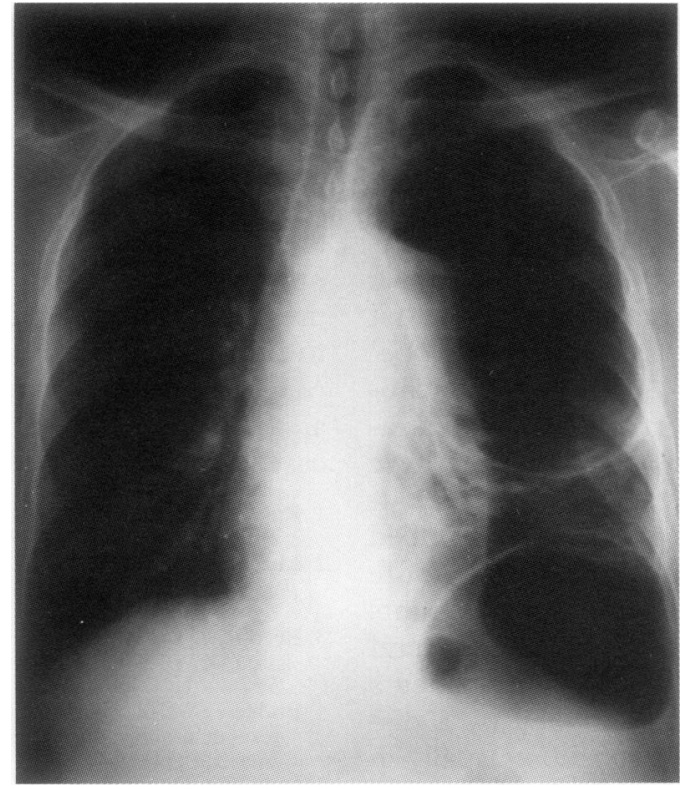

Figure 2 Chest radiograph showing further increase in the size of the bullae.

aged fluid grew Mycobacterium tuberculosis which was fully sensitive to all three drugs. Pyrazinamide was discontinued after two months.

One month after the start of chemotherapy the cough and fever had disappeared but the chest radiograph revealed two small bullae in the left lung (fig 1). Follow up chest radiography showed a steady increase in the size of the bullae at two and four months. Six months after the start of chemotherapy the left hemithorax was almost totally replaced by a giant bulla with a shift of the mediastinum to the right (fig 2). Antituberculosis drugs were continued for a total of nine months; two months after the start of chemotherapy the sputum was negative on smear and culture.

One year after the start of chemotherapy he developed hoarseness and a second fibreoptic bronchoscopic examination showed that the left vocal cord was paralysed and there was $90 \%$ stenosis of the left main bronchus with irregular mucosa. Several endobronchial biopsy specimens were taken and showed chronic nonspecific inflammation with no malignancy or granulomas.
A computed tomographic (CT) scan showed the left lung to be totally replaced by the bulla. Mediastinal windows showed no mediastinal lymphadenopathy. He was followed up for two years with no change on the chest radiograph or in spirometric values. The paralysis of the left recurrent laryngeal nerve persisted and the patient remained breathless on moderate exertion.

\section{Discussion}

Small cystic lesions of rapid onset (pneumatocoeles) are a rare but well documented complication of tuberculosis. ${ }^{12}$ However, only 26 previous reports of bullae complicating tuber- ? culosis were found, and in those reports the bullae regressed partially or completely on control of the infection.

The formation of bullae was first recorded of after the advent of chemotherapy for tuber- $\rightarrow$ culosis 45 years ago, and previous workers have $\vec{N}$ attributed this to the "lytic" effect of isoniazid. ${ }^{3}$ "

Diabetes, which can exacerbate lung cavi- 옥 tation in tuberculosis, ${ }^{45}$ might have con- $\vec{\overrightarrow{ }}$ tributed to the two unique features of our case: the large size and the lack of regression of the bulla. We do not believe the paralysis of $\overrightarrow{\mathscr{C}}$ the recurrent laryngeal nerve was related to the presence of the bulla, but was probably the result of mediastinal tuberculous lymphadenopathy which had resolved by the time the CT scan was performed and is a documented cause for this paralysis. ${ }^{67}$

1 Alarcon DG. Regressive giant bullous emphysema in tuberculosis of adults. Dis Chest 1955;27:31-43.

2 Madden RE, Viswanathan TK, Verghese KP. Solitary pneumatocele in childhood tuberculosis. Ann Thorac Surg 1968;5:347-51.

3 Purriel P, Muras O, Ardaa R. Chemical casetomy with iso- 0 niazid with appearance of mechanical defects (pneumatic changes). Thorax 1953;2:109.

4 Hendy $M$, Stableforth $D$. The effect of established diabetes mellitus on the presentation on infiltrative pulmonary tuberculosis in the immigrant Asian community of an inner tuberculosis in the immigrant Asian community of an inner city area of the United Kingdom. Br $\mathcal{F}$ Dis Chest 1983,77:

5 Ikezoe J, Takeuchi N, Jonkoh T, Kohno N, Tomiyama N, $\mathrm{O}$ Kozuka T, et al. CT appearance of pulmonary tuberculosis in diabetic and immunocompromised patient: comparison

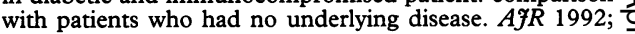
159:1175-9.

6 Fowler RW, Hetzel MR. Tuberculous mediastinal lymph- N adenopathy can cause left vocal cord paralysis. $B M \mathcal{F} 1983$; 286:1562.

7 Gupta SK. The syndrome of spontaneous laryngeal palsy in pulmonary tuberculosis. $\mathcal{f}$ Laryngol Otol 1960;74:166-73. 